\title{
Determining the Optimal Source Points in MFS by Minimizing an Energy Gap Functional for 3D Laplace Operator
}

\author{
M. Sajjadmanesh $\mathbb{D}^{1},{ }^{1}$ S. Shakib Khanghah, ${ }^{1}$ and H. Aydi $\mathbb{D}^{2,3}$ \\ ${ }^{1}$ Faculty of Basic Science, University of Bonab, Bonab, Iran \\ ${ }^{2}$ Université de Sousse, Institut Supérieur d'Informatique et des Techniques de Communication, H. Sousse 4000, Tunisia \\ ${ }^{3}$ China Medical University Hospital, China Medical University, Taichung 40402, Taiwan
}

Correspondence should be addressed to M. Sajjadmanesh; m.sajjadmanesh@ubonab.ac.ir and H. Aydi; hassen.aydi@isima.rnu.tn

Received 10 October 2020; Revised 24 November 2020; Accepted 30 November 2020; Published 21 December 2020

Academic Editor: Kottakkaran Sooppy Nisar

Copyright (c) 2020 M. Sajjadmanesh et al. This is an open access article distributed under the Creative Commons Attribution License, which permits unrestricted use, distribution, and reproduction in any medium, provided the original work is properly cited.

\begin{abstract}
In this paper, an extended version of the method of minimizing an energy gap functional for determining the optimal source points in the method of fundamental solutions (MFS) is applied to the 3D Laplace operator subject to the Dirichlet and Neumann boundary conditions. As we know, the MFS is a more popular meshless method for solving boundary or initial-boundary value problems due to its simplicity and high accuracy. However, the accuracy of the MFS depends strongly on the distribution of the source points. Finally, some of the numerical experiments are carried out to express the simplicity and effectiveness of the presented method.
\end{abstract}

\section{Introduction}

In the last decades, the fixed point results have been improved and generalized in different directions for solving boundary value problems (see [1-5]), even the fixed point theorems have been extended for establishing the existence of solutions for fractional differential equations, as well as for integral equations (see $[6,7])$. These results have been usually obtained by analytic techniques and various fixed point theorems. The method of a fundamental solution (MFS) is one of the meshless numerical methods, which was first proposed by Kupradze and Aleksidze [8] in 1964. This method approximates the solution of a boundary value problem (BVP) by a linear combination of fundamental solutions of the governing partial differential operator, provided that the fundamental solution is known. The MFS is a very popular boundary meshless method due to its simplicity and high accuracy. However, this method has a serious drawback that the resulting algebraic equations system may be highly ill-conditioned when the number of source points is increased [9], or when the distances of source points are increased [10]. The method of minimizing an energy gap functional is an effective and efficient way to determine the location of source points, which was first proposed by Wang et al. in [11] for a mixed boundary value problem of $2 \mathrm{D}$ Laplace equation. In this paper, we extend the same recent method for the 3D Laplace equation with Dirichlet and Neumann boundary conditions. Finally, several numerical examples are provided to illustrate the efficiency and simplicity of the method.

This paper is organized as follows. In the next section, the MFS for the 3D Laplace operator is described. In Section 3, the energy gap functional is constructed and finally, in Sections 4 and 5, the numerical algorithm and some of the numerical experiments, respectively, are carried out for proposed method.

\section{Statement of Problem and the MFS}

Consider the mixed boundary value problem of the $3 \mathrm{D}$ Laplace equation in spherical coordinates:

$$
\begin{aligned}
\Delta u(r, \theta, \varphi) & =0, \text { in } \Omega, \\
u(\rho, \theta, \varphi) & =f(\theta, \varphi), \text { on } \Gamma_{D},
\end{aligned}
$$




$$
\frac{\partial u}{\partial n}(\rho, \theta, \varphi)=g(\theta, \varphi), o n \Gamma_{N},
$$

where $\Omega \subset \mathbb{R}^{3}$ is a bounded simply connected domain with the boundary $\partial \Omega=\Gamma_{D} \cup \Gamma_{N}$ :

$$
\begin{aligned}
\Gamma_{D} & =\{r=\rho(\theta, \varphi), 0 \leq \theta \leq \alpha \pi, 0 \leq \varphi \leq \pi\}, \Gamma_{N} \\
& =\{r=\rho(\theta, \varphi), \alpha \pi<\theta<2 \pi, 0 \leq \varphi \leq \pi\} .
\end{aligned}
$$

The function $\rho(\theta, \varphi)$ is used to describe the boundary shape, the constant $\alpha \in(0,2)$ is given, and $n$ is the outward unit normal at $\partial \Omega$.

In the MFS, the numerical solution of $u$ at the field point $z=r(\cos (\theta) \sin (\varphi), \sin (\theta) \sin (\varphi), \cos (\varphi)) \quad$ can be expressed as a linear combination of the fundamental solutions $U\left(z, s_{j}\right)$ :

$$
u(z)=\sum_{j=1}^{n_{s}} c_{j} U\left(z, s_{j}\right)
$$

where the source points

$$
s_{j}=R_{j}\left(\cos \left(\theta_{j}\right) \sin \left(\varphi_{j}\right), \sin \left(\theta_{j}\right) \sin \left(\varphi_{j}\right), \cos \left(\varphi_{j}\right)\right), 1 \leq j \leq n_{s}
$$

uniformly located on the complementary set $\Omega^{c}$ with

$$
R_{j}=D+\rho\left(\theta_{j}, \varphi_{j}\right), 1 \leq j \leq n_{s}
$$

and $D$ is an unknown offset to be determined such that the error of the numerical solution obtained by the MFS is minimized.

The fundamental solution $U\left(z, s_{j}\right)$ of $3 \mathrm{D}$ Laplace equation (1) is given by [12]

$$
U\left(z, s_{j}\right)=\frac{1}{4 \pi r_{j}}, r_{j}=\left\|z-s_{j}\right\| .
$$

By collecting $n_{c}$ points

$$
z_{i}=\rho\left(\theta_{i}, \varphi_{i}\right)\left(\cos \left(\theta_{i}\right) \sin \left(\varphi_{i}\right), \sin \left(\theta_{i}\right) \sin \left(\varphi_{i}\right), \cos \left(\varphi_{i}\right)\right), 1 \leq i \leq n_{c}
$$

uniformly located on the boundary $\partial \Omega$ and satisfying the boundary conditions (2) and (3) and considering (5), a linear equation system can be achieved to determine the coefficients $c_{j}$.

\section{Energy Gap Functional}

To determine the optimal source points, we have the following result which is an extension of Theorem 1 in [11] to 3D Laplace operator.
Theorem 1. The energy gap functional with respect to the mixed BVP (1)-(3) is given by

$$
\begin{aligned}
\mathscr{G}= & \iint_{\Gamma_{D}} u_{n}(\rho(\theta, \varphi), \theta, \varphi) f(\theta, \varphi) d \sigma \\
& +\iint_{\Gamma_{N}} u(\rho(\theta, \varphi), \theta, \varphi) g(\theta, \varphi) d \sigma \\
& -\iiint_{\Omega}\|\nabla u(r, \theta, \varphi)\|^{2} d s=0,
\end{aligned}
$$

where $d \sigma=|(\partial r / \partial \theta) \times(\partial r / \partial \varphi)| d \theta d \varphi$ and $d s=\rho^{2} \sin (\varphi) d \rho$ $d \theta d \varphi$.

Proof. Multiplying both sides of Eq. (1) by $u$ and integrating it over domain $\Omega$, we can derive

$$
\iiint_{\Omega} u \Delta u d s=0
$$

which can be rewritten as

$$
\iiint_{\Omega}\left[\nabla \cdot(u \nabla u)-\|\nabla u\|^{2}\right] d s=0
$$

Applying the Gaussian divergence theorem [13] on Eq. (12) yields that

$$
\iiint_{\Omega}\|\nabla u\|^{2} d s=\iint_{\partial \Omega}(u \nabla u) \cdot n d \sigma
$$

where $d \sigma=|(\partial r / \partial \theta) \times(\partial r / \partial \varphi)| d \theta d \varphi$, and $n$ is the outward unit normal at $\partial \Omega=\Gamma_{D} \cup \Gamma_{N}$. Finally, by using the boundary conditions (2) and (3) in Eq. (13), the energy gap functional (10) can be derived, and the proof is completed.

The numerical solution $u$ of the problem (1)-(3) in MFS usually does not satisfy the energy gap in Eq. (10), i.e., $\mathscr{G} \neq$ 0 . Then, we attempt to minimize the energy gap $\mathscr{G}$ by finding the optimal value of $D$, i.e.,

$$
\min _{D \in\left[D_{1}, D_{2}\right]}|\mathscr{G}|
$$

where $\left[D_{1}, D_{1}\right]$ is an interval of which we attempt to seek the optimal value of $D$.

\section{A Numerical Algorithm}

Suppose that $M$ is the number of subintervals of $\left[D_{1}, D_{2}\right]$ which we attempt to minimize the energy gap functional $\mid \mathscr{G}$ I in Eq. (10). Moreover, $n_{c}$ and $n_{s}$ are the number of collocation and source points, respectively. For $L=0,1, \cdots, M$, we do the following steps:

(I) Let

$$
D_{L}=D_{1}+L \frac{D_{2}-D_{1}}{M}
$$


and compute

$$
\begin{aligned}
s_{j}= & \left(D_{L}+\rho\left(\theta_{j}, \varphi_{j}\right)\right)\left(\cos \left(\theta_{j}\right) \sin \right. \\
& \left.\cdot\left(\varphi_{j}\right), \sin \left(\theta_{j}\right) \sin \left(\varphi_{j}\right), \cos \left(\varphi_{j}\right)\right), \\
U\left(z_{i}, s_{j}\right)= & \frac{1}{4 \pi}\left\{\left(x_{i}^{c}-x_{j}^{s}\right)^{2}+\left(y_{i}^{c}-y_{j}^{s}\right)^{2}\right. \\
& \left.+\left(z_{i}^{c}-z_{j}^{s}\right)^{2}\right\}^{-1 / 2} 1 \leq i \leq n_{c}, 1 \leq j \leq n_{s},
\end{aligned}
$$

where $z_{i}=\left(x_{i}^{c}, y_{i}^{c}, z_{i}^{c}\right)$ and $s_{j}=\left(x_{j}^{s}, y_{j}^{s}, z_{j}^{s}\right)$ are, respectively, the collocation and source points uniformly disturbed on $\partial \Omega$ and $\partial D$ with

$$
\begin{aligned}
\partial D=\{ & \left(D_{L}+\rho(\theta, \varphi)\right)(\cos (\theta) \sin (\varphi), \sin \\
& \cdot(\theta) \sin (\varphi), \cos (\varphi)), 0 \leq \theta<2 \pi, 0 \leq \varphi \leq \pi\}
\end{aligned}
$$

(II) Inserting the collocation and source points $z_{i}, s_{j}$ into Eq. (5) and enforcing it to satisfy the boundary conditions (2) and (3), the following linear equations system can be derived

$$
A c=b,
$$

where $c=\left(c_{1}, c_{2}, \cdots, c_{n_{s}}\right)^{T}$, and the $n_{c} \times n_{s}$ matrix $A$ is in general ill-conditioned

The conjugate gradient method (CGM) can be used to solve the overdetermined system (18) as follows:

(III) Let

$$
R_{0}=\frac{1}{n_{s}} \sum_{k=1}^{n_{s}}\left\{\sum_{j=1}^{n_{c}} a_{j k}^{2}\right\}^{1 / 2}
$$

where $a_{j k}$ is the $j k^{t h}$ component of the coefficient matrix $A$

Compute

$$
s(:, k)=\frac{R_{0}}{N(:, k)},
$$

where $N(:, k)$ denotes the norm of the $k^{\text {th }}$ column of matrix $A$ and let

$$
B=A P,
$$

where $P=\operatorname{diag}(s)$ denotes the diagonal matrix with components $s_{1}, s_{2}, \cdots, s_{n_{s}}$. Then, the following overdetermined algebraic system with well-conditioned coefficient matrix $B$ can be derived:

$$
B c_{1}=b \text {, }
$$

TABLE 1: Comparing the accuracy with different $n_{c}$ for Example 2.

\begin{tabular}{lccc}
\hline$n_{c}$ & ME & RMSE & Minimum energy gap \\
\hline 50 & $9.2605 \times 10^{-3}$ & $4.237 \times 10^{-3}$ & $1.477 \times 10^{-2}$ \\
200 & $8.1481 \times 10^{-4}$ & $2.5906 \times 10^{-4}$ & $1.6706 \times 10^{-3}$ \\
450 & $7.3568 \times 10^{-4}$ & $2.1993 \times 10^{-4}$ & $1.6488 \times 10^{-3}$ \\
\hline
\end{tabular}

TABle 2: Comparing the accuracy with different $n_{c}$ for Example 3.

\begin{tabular}{lccc}
\hline$n_{c}$ & ME & RMSE & Minimum of energy gap \\
\hline 50 & $1.3657 \times 10^{-3}$ & $5.0442 \times 10^{-4}$ & $1.634 \times 10^{-3}$ \\
200 & $4.5631 \times 10^{-4}$ & $1.5347 \times 10^{-4}$ & $3.159 \times 10^{-4}$ \\
450 & $3.0346 \times 10^{-4}$ & $1.0369 \times 10^{-4}$ & $1.864 \times 10^{-4}$ \\
\hline
\end{tabular}

where the all of rows or columns of matrix $B$ have the same norm $R_{0}$.

(IV) Let

$$
D=B^{T} B, b_{1}=B^{T} b
$$

Then, the following linear equation system with the positive definite matrix $D$ can be obtained:

$$
D c_{1}=b_{1}
$$

(V) Solve $c_{1}$ from system (24) by the CGM and denote it by $c_{1 L}$ and let $c_{L}=s \cdot c_{1 L}$, where $s$ is a column matrix $s=\left(s_{1}, s_{2}, \cdots, s_{n_{s}}\right)^{T}$, and ". denotes the inner product

(VI) Compute $|\mathscr{G}|$ by inserting Eq. (5) for $u$ into Eq. (10)

(VII) Consider the criterion of stopping and if it is achieved, then $D=D_{L}$ is the optimal offset and inserting $D$ and $c=c_{L}$, the optimal solution $u$ can be obtained

\section{Numerical Experiments}

In this section, we give two examples of mixed boundary value problems to check the effectiveness of the presented method. The implementation of the algorithm is based on the MATLAB software. We denote the analytical and numerical solutions by $u_{\text {ana }}$ and $u_{\text {num }}$, respectively. Define the maximum error (ME) and the root-mean-square-error (RMSE) as follows:

$$
\begin{gathered}
\operatorname{ME}(u)=\max \left|u_{\text {ana }}\left(x_{l}\right)-u_{\text {num }}\left(x_{l}\right)\right|, l=1,2, \cdots, n_{c}, \\
\operatorname{RMSE}(u)=\left\{\frac{1}{n_{c}} \sum_{l=1}^{n_{c}}\left(u_{\text {ana }}\left(x_{l}\right)-u_{\text {num }}\left(x_{l}\right)\right)^{2}\right\}^{1 / 2} .
\end{gathered}
$$

In Tables 1 and 2, we list the minimum energy gap $|\mathscr{G}|$, $\mathrm{ME}$, and RMSE for different values of $n_{c}$ with $n_{s}=50$. The curves of errors ME and RMSE and also, the optimal value 


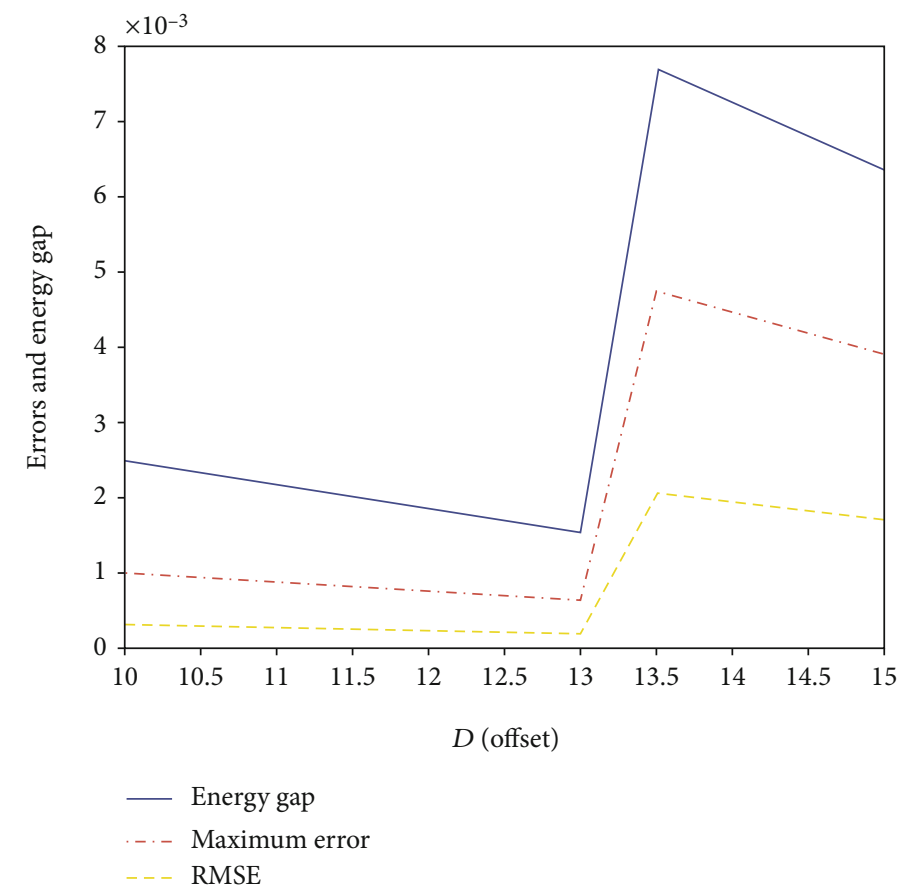

FIgURE 1: Determining the optimal offset in the MFS by minimizing the energy gap for Example 2.

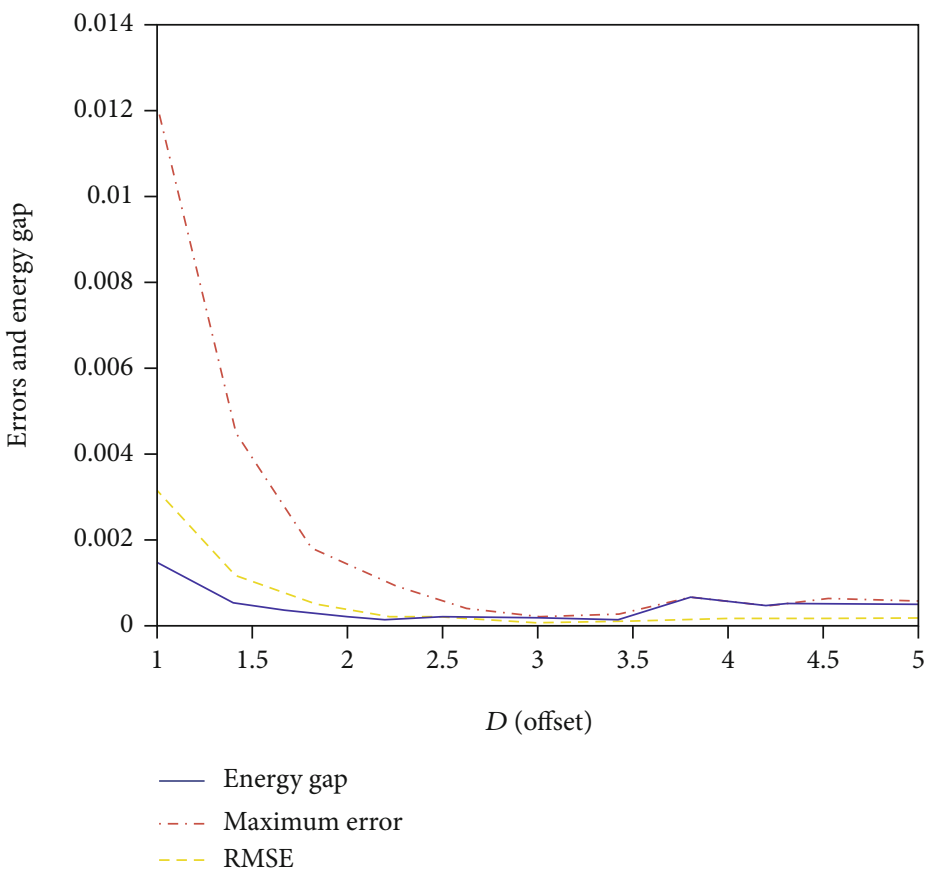

FIGURE 2: Determining the optimal offset in the MFS by minimizing the energy gap for Example 3.

$D_{o p t}$ in $\left[D_{1}, D_{2}\right]$, from the minimization problem (14) with $\mathscr{G}$ given by Eq. (10) are given in Figures 1 and 2 to show the accuracy and efficiency of the proposed method.

Example 2. Consider the mixed boundary value problem (1)(3) on the unit sphere

$$
\Omega=\{(r, \theta, \varphi) ; 0<r<1,0 \leq \theta<2 \pi, 0 \leq \varphi \leq \pi\},
$$

with

$$
\begin{aligned}
& \Gamma_{D}=\{(1, \theta, \varphi) ; 0 \leq \theta \leq \pi, 0 \leq \varphi \leq \pi\}, \\
& \Gamma_{N}=\{(1, \theta, \varphi) ; \pi<\theta<2 \pi, 0 \leq \varphi \leq \pi\} .
\end{aligned}
$$

The exact solution is given by $u\left(x_{1}, x_{2}, x_{3}\right)=1 / 2\left(x_{1}^{2}+x_{2}^{2}\right)$ $-x_{3}^{2}$. Here, $\left[D_{1}, D_{2}\right]=[10,15]$ is an interval we attempt to 
search the optimal value of $D$, of which $D_{o p t}=13$ is obtained as shown in Figure 1.

Example 3. Consider the mixed boundary value problem (1)(3) on the bumpy sphere

$$
\Omega=\left\{(r, \theta, \varphi) ; r=\rho\left(1+\frac{1}{6} \sin (\varphi)\right), 0 \leq \rho<1,0 \leq \theta<2 \pi, 0 \leq \varphi \leq \pi\right\},
$$

with

$$
\begin{aligned}
& \Gamma_{D}=\{x \in \partial \Omega ; 0 \leq \theta(x) \leq \pi, 0 \leq \varphi(x) \leq \pi\}, \\
& \Gamma_{N}=\{x \in \partial \Omega ; \pi<\theta(x)<2 \pi, 0 \leq \varphi(x) \leq \pi\} .
\end{aligned}
$$

The exact solution is given by $u\left(x_{1}, x_{2}, x_{3}\right)=x_{1} x_{2}+x_{1} x_{3}$ $+x_{2} x_{3}$. The optimal value of $D$ in $\left[D_{1}, D_{2}\right]=[1,5]$ which is $D_{\text {opt }}=3.4$ as shown in Figure 2 .

\section{Conclusion}

In this study, the mixed boundary value problem, which consists of determining the optimal source points in MFS, has been investigated by minimizing an energy gap functional for 3D Laplace operator subject to the Dirichlet and Neumann boundary conditions. The method was first proposed by Wang et al. in [11] for a mixed boundary value problem involving the 2D Laplace equation, and we extended it for the $3 \mathrm{D}$ case. Inserting the collocation and source points in MFS and enforcing the boundary conditions yield to a system of linear equations with general ill-conditioned coefficient matrix which can be solved by one of the regularization methods such as the conjugate gradient method (CGM). Finally, the obtained numerical solutions via the MFS can be placed in an energy gap functional until its minimum value is obtained for the appropriate source points. Two examples for the unit spherical and the bumpy spherical boundaries have been considered. The numerical results show that minimizing of an energy gap functional is a simple and efficient method for determining source points in the MFS.

\section{Data Availability}

The data used to support the findings of this study are available from the corresponding author upon request.

\section{Conflicts of Interest}

The author(s) declare(s) that they have no conflicts of interest.

\section{References}

[1] H. Aydi, M. Jleli, and B. Samet, "On positive solutions for a fractional thermostat model with a convexconcave source term via $\psi$-Caputo fractional derivative," Mediterranean Journal of Mathematics, vol. 17, no. 1, p. 16, 2020.
[2] P. Debnath and H. M. Srivastava, "Global optimization and common best proximity points for some multivalued contractive pairs of mappings," Axioms, vol. 9, no. 3, p. 102, 2020.

[3] Z. Ma, A. Asif, H. Aydi, S. Ullah Khan, and M. Arshad, "Analysis of F-contractions in function weighted metric spaces with an application," Open Mathematics, vol. 18, no. 1, pp. 582-594, 2020.

[4] S. A. Harisa, M. A. A. Khan, F. Mumtaz et al., "Shrinking Cesáro means method for the split equilibrium and fixed point problems in Hilbert spaces," Advances in Difference Equations, vol. 1,19 pages, 2020 .

[5] M. Berzig, S. Chandok, and M. S. Khan, "Generalized Krasnoselskii fixed point theorem involving auxiliary functions in bimetric spaces and application to two-point boundary value problem," Applied Mathematics and Computation, vol. 248, pp. 323-327, 2014.

[6] P. Debnath and H. M. Srivastava, "New extensions of Kannan's and Reich's fixed point theorems for multivalued maps using Wardowski's technique with application to integral equations," Symmetry, vol. 12, no. 7, p. 1090, 2020.

[7] R. K. Sharma and S. Chandok, "Multivalued problems, orthogonal mappings, and fractional integro-differential equation," Journal of Mathematics, vol. 2020, Article ID 6615478, 8 pages, 2020.

[8] V. D. Kupradze and M. A. Aleksidze, "The method of functional equations for the approximate solution of certain boundary value problems," USSR Computational Mathematics and Mathematical Physics, vol. 4, no. 4, pp. 82-126, 1964.

[9] G. Fairweather and A. Karageorghis, "The method of fundamental solutions for elliptic boundary value problems," Advances in Computational Mathematics, vol. 9, no. 1/2, pp. 69-95, 1998.

[10] C. S. Chen, H. A. Cho, and M. A. Golberg, "Some comments on the ill-conditioning of the method of fundamental solutions," Engineering Analysis with Boundary Elements, vol. 30, no. 5, pp. 405-410, 2006.

[11] F. Wang, C. S. Liu, and W. Qu, "Optimal sources in the MFS by minimizing a new merit function: energy gap functional," Applied Mathematics Letters, vol. 86, pp. 229-235, 2018.

[12] V.S. Vladimirov, Equations of Mathematical Physics, Mir Publishers, Moscow, 1981.

[13] R. A. Adams and C. Essex, Calculus: A complete course, Pearson Canada Inc., Toronto, Canada, 2010. 